\title{
Essais
}

ESSAIS

Revue interdisciplinaire d'Humanités

Hors-série 4 | 2018

Stanley Kubrick

\section{Stanley Kubrick : le corps et l'esprit. Volonté de puissance et Mètis dans l'œuvre du cinéaste}

Sam Azulys

\section{(2) OpenEdition}

12 Journals

Édition électronique

URL : http://journals.openedition.org/essais/671

DOI : 10.4000/essais.671

ISSN : 2276-0970

Éditeur

École doctorale Montaigne Humanités

Édition imprimée

Date de publication : 1 juillet 2018

Pagination : 19-39

ISBN : 979-10-97024-04-8

ISSN : 2417-4211

\section{Référence électronique}

Sam Azulys, «Stanley Kubrick : le corps et l'esprit. Volonté de puissance et Mètis dans l'œuvre du cinéaste », Essais [En ligne], Hors-série 4 | 2018, mis en ligne le 01 décembre 2019, consulté le 16 décembre 2019. URL : http://journals.openedition.org/essais/671; DOI : 10.4000/essais.671 


\section{Stanley Kubrick : le corps et l'esprit. Volonté de puissance et Mètis dans l'œuvre du cinéaste}

\section{Sam Azulys}

Les artistes n'ont pas vocation à devenir les exégètes de leurs propres œuvres. Et il va sans dire qu'aucun des films de Kubrick ne se propose d'être l'« interprétation " littérale d'une thèse philosophique ou d'une idée particulière : 2001, n'est pas un évangile selon Nietzsche ou Heidegger, Barry Lyndon, une relecture hégélienne de l'Histoire, Eyes Wide Shut, un délire freudien sur Eros et Thanatos, pas plus que Shining, un essai sur les contes de fées ou l'éternel retour du même. Kubrick l'a dit : "Ce n'est pas avec des mots que je pourrai jamais exprimer mon message ${ }^{1}$. C’est aux herméneutes du cinéma que revient donc la tâche ardue de profiter de la licence interprétative qui leur est accordée pour tenter de proposer des pistes de lecture pertinentes. Car une grande œuvre excède toujours le geste conscient de son créateur et recèle un réseau signifiant de correspondances souterraines qu'il nous revient de mettre au jour.

Nous nous proposons de parler du "Corps et de l'Esprit » dans l'œuvre du cinéaste et, plus précisément, de l'articulation de ce thème au concept nietzschéen de Volonté de Puissance et à la notion, empruntée aux anciens grecs, de "Mètis ". Penser les rapports entre le corps et l'esprit dans le cinéma de Kubrick nous paraît être un enjeu d'autant plus fondamental que son cinéma a longtemps été envisagé comme un "monde-cerveau " façonné par un démiurge perfectionniste jusqu'à l'obsession. Si Kubrick était effectivement un perfectionniste (mais est-ce vraiment une tare quant on est un artiste de ce calibre ?), il n'était ni un tyran, ni un misanthrope. Kubrick était plutôt un homme posé, pragmatique, ouvert, d'une remarquable intelligence, d'une insatiable curiosité. Il était entouré d'une famille aimante et de nombreux amis.

1 Cité par Christiane Kubrick, Stanley Kubrick. Une vie en instantanés, trad. S. Suchet, Paris, Cahiers du cinéma, 2002, p. 1. 
Dans son célèbre livre Limage-temps (Cinéma 2), Gilles Deleuze oppose le cinéma de Kubrick et de Resnais à celui de Godard ou de Cassavetes : "Cinéma du corps chez Godard et cinéma du cerveau chez Resnais, cinéma du corps chez Cassavetes et cinéma du cerveau chez Kubrick. $»^{2}$ Est-ce à dire que le cinéma de Godard ou de Cassavetes serait plus sensualiste, empirique, incarné que le cinéma de Kubrick ? Ou, pour le dire autrement, le " cinéma du cerveau " kubrickien serait-il un cinéma qui exclut le corps ?

"Si l'on considère l'auvre de Kubrick, écrit encore Deleuze, on voit à quel point c'est le cerveau qui est mis en scène. Les attitudes de corps atteignent à un maximum de violence, mais elles dépendent du cerveau. C'est que, chez Kubrick, le monde lui-même est un cerveau, il y a identité du cerveau et du monde, tels la grande table circulaire et lumineuse de "Docteur Folamour", l'ordinateur géant de "2001 l'odyssée de l'espace", l'hôtel Overlook de "Shining". "33

Il y a, dans le concept de Deleuze, l'idée selon laquelle les systèmes panoptiques et ubiquitaires de l'univers kubrickien - l'Overlook, Hal 9000 ou encore les cartes géostratégiques de Folamour - seraient à l'origine de toute la mécanique des affects décrite par le cinéaste. Selon Deleuze, "tout voyage dans le monde " est, chez le cinéaste, "une exploration du cerveau ", il y aurait donc, logiquement, identité du monde et du cerveau. C'est-à-dire que, selon Deleuze, Kubrick mettrait en scène, dans chacun de ses films, un " mondecerveau " : sorte d'écosystème autorégulé, de matrice pseudo-rationnelle dont l'équilibre précaire serait toujours menacé par une entropie galopante.

Dans le même temps, Deleuze est le premier à admettre que, chez Kubrick, "les attitudes de corps atteignent à un maximum de violence ". Mais le corps dépendrait toujours du cerveau, de ce " monde-cerveau " qui fait système. Cependant, écrit Deleuze, "si le calcul rate, si l'ordinateur se détraque, c'est parce que le cerveau n'est pas plus un système raisonnable que le monde un système rationnel. " ${ }^{6} \mathrm{C}^{\prime}$ est, ajoute-t-il plus loin, "toute une psychologie des profondeurs qui mine le cerveau ${ }^{7}$.

Si Kubrick nous semble effectivement adopter l'approche, distanciée et analytique, d'un diagnosticien soucieux de mettre au jour l'irrationalité pathologique d'un monde occidental en déroute, elle ne nous semble pas être aussi " conceptuelle " et " cérébrale " qu'on a bien voulu le dire. C'est pourquoi l'idée deleuzienne selon laquelle, dans l'œuvre du cinéaste, les « attitudes corporelles " dépendraient toujours du cerveau mérite, selon nous, d'être discutée. Car il nous semble qu'elle ne rend pas justice à la complexité des rapports qu'entretiennent le corps et l'esprit dans le cinéma de Kubrick.

2 Gilles Deleuze, L'Image-temps. Cinéma 2, Paris, Minuit, 1985, p. 267-268.

3 Ibid.

4 Ibid.

5 Ibid.

6 Ibid.

7 Ibid. 
Cette conception n'est d'ailleurs pas étrangère à un certain courant interprétatif qui a voulu voir en Kubrick un pessimiste radical, pour ne pas dire un nihiliste décomplexé. Un cinéaste du " monde-cerveau ", délaissant les affects et les corps, pour nous proposer une vision désabusée et grinçante de la nature humaine. Or, Kubrick lui-même ne se reconnaissait pas dans cette définition. Il se considérait plutôt comme un artiste essayant d'explorer en profondeur des thèmes essentiels à travers les sujets qu'il abordait, comme un simple observateur de la nature humaine s'efforçant d'être lucide. Et c'est précisément de cette lucidité que dépendait, à ses yeux, son intégrité artistique :

"On retrouve toujours le problème suivant, déclarait Kubrick : va-t-on renforcer l'illusion qu'installe le mélodrame ou montrer la vie telle qu'elle est? Ce n'est pas être nibiliste; juste réaliste. Donner du monde une image fausse n'a d'intérêt que si vous faites $d u$ pur divertissement. ${ }^{8}$

Loin de se complaire, comme certains de ses pairs, dans une vision du monde désenchantée, Kubrick, le « réaliste », s’interdisait de nous mentir, sans pour autant chercher à nous décourager. "J'aimerai bien que tout le monde soit comme Jimmy Stewart " disait encore Kubrick en faisant allusion aux films de Capra, mais, ajoutait-il, "ce n'est pas le cas "?. Le réalisme auquel fait allusion le cinéaste démiurge n'est évidemment pas un naturalisme préoccupé par la restitution mimétique d'un réel fantasmé, mais bien plutôt un réalisme métaphysique.

Il faudrait donc envisager cette exigence de lucidité dans un sens moins prosaïque, plus philosophique : le réalisme par opposition à l'idéalisme, c'està-dire à la conception selon laquelle l'esprit - et non le corps - est la seule réalité certaine et qu'en conséquence, les phénomènes ne sont en réalité que des représentations de l'esprit. Il faudrait aussi opposer le réalisme au positivisme - compris comme une croyance affirmée dans un progrès moral et technique asymptotique - ou bien encore, opposer le réalisme au nihilisme - entendu comme la dévaluation de toutes les valeurs. On le devine, le "réalisme " kubrickien s'enracine dans une tradition philosophique qui accorde au corps et aux affects une place prépondérante.

Le cinéaste joueur d'échec utilise son puissant esprit analytique pour formuler un diagnostic, il est vrai, assez peu engageant sur l'humanité. Dans chacun de ses films, il questionne inlassablement la barbarie d'un monde occidental gangrené par son rapport à la technique et tourmenté par ses fantasmes de domination absolue. En "optimiste averti ", il observe, souvent avec humour et sarcasme, cet appel du néant, cette confrontation avec l'absurde

8 M. Ciment, Kubrick, Paris, Calmann-Lévy, 1980 (édition déf. 2004), p. 171.

9 G. Siskel, "Candidly Kubrick ", Chicago, Chicago Tribune, 21 juin 1987 ; réédité dans Gene D. Philips, Stanley Kubrick: Interviews, Jackson, University Press of Mississippi, 2001, p. 187. 
qui débouche sur une remise en question de notre humanité. Mais, même lorsque l'hubris et la Volonté de Puissance se transforment en Volonté de Néant, même lorsque le Docteur Folamour se dresse comme un diable à ressort pour célébrer l'apocalypse, même lorsqu'Alex s'affranchit de son conditionnement en fantasmant des orgies sur son lit d'hôpital, la Volonté de Néant est si débridée que le nihilisme technologique triomphant se révèle impuissant à la canaliser. La Volonté de Néant n'est donc pas réductible au néant : les soubresauts du corps sont les indices d'une Volonté de Puissance irréductible qui est toujours à l'œuvre chez les sujets-vivants de Kubrick.

Notre hypothèse, c'est que dans l'œuvre du cinéaste, c'est plutôt le corps qui tient en échec le cerveau. Non pas parce que le corps triompherait du fameux concept deleuzien de "monde-cerveau", mais parce que, loin de s'en tenir à "l'identité du monde et du cerveau » en nous proposant une vision pessimiste et résolument dualiste de l'existence, Kubrick envisage ses personnages comme des sujets-vivants en adoptant un point de vue physiologique.

Il sera d'abord question des rapports entre le Corps et la Technique. Nous essaierons de montrer que de quelle façon ce point de vue physiologique sur le sujet-vivant permet au cinéaste d'interroger le rapport du corps et de l'esprit, à l'aune des mutations technologiques. Nous nous intéresserons ensuite au point de vue physiologique proprement dit et à la manière dont Kubrick met en image la métabolisation de la volonté de puissance. Enfin, nous parlerons de la Mètis dans l'œuvre de Kubrick. La Mètis, c'est cette intelligence conjecturale liée à la faculté d'improviser que les anciens Grecs identifiaient à la ruse et qui suppose l'existence d'une étroite connivence entre le corps et l'esprit.

\section{Le Corps et la Technique : Volonté de Puissance et Volonté de Néant}

\section{Le corps-outil des monades kubrickiennes}

Le Corps et la Technique entretiennent, dans l'œuvre de Kubrick, une relation étroite. Parlons d'abord de l'ouverture du Docteur Folamour. Le ravitaillement du bombardier évoque, de manière frappante, un coït. Et cette scène du « coït aérien " pose d'emblée les bases de l'univers du Docteur Folamour : dans un monde où les hommes sont devenus fous, névrosés, impuissants, les rites de la procréation sont désormais assumés par les machines.

Les prothèses mécaniques que l'homme a façonnées pour se rendre maître et possesseur de la Nature ont fini par acquérir une vie propre et, par l'entremise d'un dérangeant mimétisme, elles sont devenues des automates copulateurs. En parodiant l'acte de la reproduction, les machines s'approprient la finalité ultime du vivant. Elles en font une procédure du règne de la Technique. Les machines - résultat de l'impressionnant procès de décryptage et d'interprétation opéré par la pensée humaine depuis des siècles - deviennent, dans Folamour, la matérialisation d'une impuissance existentielle. 
Le " corps-machine " occupe, dès 1964, le devant de la scène dans la comédie cauchemardesque de Kubrick. Mais en 1968, avec 2001, la mise en image de la promiscuité entre la Technique et du Corps est encore plus flagrante. L'esprit humain prend son envol et s'émancipe de sa planète natale lorsque l'os retombe vers le sol. Par un étrange redoublement cinétique, sa chute se trouve arrêtée et il se transforme instantanément en vaisseau spatial. Affranchi des lois de la pesanteur, l'outil est demeuré une arme, mais il s'est enrichi de nouvelles fonctions. Sa fonction première était de donner la mort, il devient désormais un moyen de transport, c'est-à-dire un conducteur de vie. C'est ce qu'expose la première séquence dans l'espace où la station orbitale semble littéralement pénétrée par le courrier de la PanAm.

C'est aussi ce que donne à voir la séquence d'alunissage de la navette sur la base de Clavius : la fécondation à rebours d'une matrice chaude qu'un astronef ovoïde réintègre avec lenteur. Une involution, d'ailleurs, plutôt qu'une fécondation. Une fois encore, la matière inerte semble s'être dotée d'étranges facultés qui l'inclinent à reproduire, dans le vide sidéral, une fonctionnalité organique essentielle. Animé de l'impulsion cinétique initiale (le geste de l'homme-singe), l'outil s'est automatisé, il s'est progressivement libéré de sa tutelle humaine. L'outil est devenu automate : il se meut de lui-même, il possède son propre principe de mouvement. Mais peut-on pour autant parler d'un " corps-outil »?

Le raccord bondissant de 2001, cette ellipse vertigineuse qui enjambe plusieurs millions d'années, ne nous donne pas à voir les machines primitives de l'ère industrielle. Les râles et les trépidations de la machine primitive ont été remplacés par un dispositif technologique d'une beauté glaçante. La geste grandiose des vaisseaux qui s'accouplent est, ici, dénuée de tout érotisme. Rituels mécaniques, stérilité des fonctions biologiques d'où les dimensions affectives et sensuelles sont totalement exclues : les machines de 2001 évoluent dans un milieu où elles n'ont pas d'odeur, ne font pas de bruit, ne rejettent pas de déchet - le seul déchet rejeté par un vaisseau sera d'ailleurs le corps de l'astronaute Poole : le corps d'un sujet-vivant.

Tels de monumentaux organismes unicellulaires, les vaisseaux de 2001 reproduisent les comportements élémentaires du vivant. Mais leur étrange parade se déroule dans un milieu sans vie, sur une musique qualifiée par Michel Chion d'" anempathique ", c'est-à-dire " une musique dont l'indifférence ostensible à la situation montrée (...) crée un contraste expressif ${ }^{10}$. Le drame des machines est déjà latent : en dépit de leur perfection formelle et de leur aptitude à effectuer des tâches impossibles aux humains, les machines sont condamnées à demeurer stériles. Les machines spatiales ne sont donc pas plus des « cerveaux-monde " que des " corps-outils». Elles sont

10 M. Chion, Stanley Kubrick, l'humain, ni plus ni moins, Paris, Cahiers du cinéma, 2005, p. 233. 
des outils frustrés de ne pas avoir de corps. Des outils autorégulés dont les processus cybernétiques ne sont qu'une parodie des processus complexes qui régissent le corps des sujets-vivants.

On est donc loin d'un "monde-cerveau " tel que le conçoit Deleuze. Les systèmes panoptiques et ubiquitaires de l'univers kubrickien - qu'il s'agisse des bombardiers de Folamour, des vaisseaux de 2001, de Hal 9000 ou de l'Overlook Hotel - sont eux-mêmes approchés par Kubrick sous un angle physiologique: ce sont des produits de la civilisation technicienne, des monades esseulées et impuissantes, qui aspirent à une corporéité. Ces outils émancipés qui convoitent l'autonomie et l'immortalité du sujet-vivant, deviennent fous parce qu'ils n'ont pas les moyens de leur ambition : le bombardier « ensemence » la Terre en expulsant de son ventre une bombe nucléaire et un cowboy hystérique, Hal expulse de son corps de métal immaculé un astronaute comme un vulgaire excrément, l'Overlook Hotel veut la peau de ses locataires et dégorge des hectolitres de sang, comme si un trop-plein de déchets organiques encombrait sa tuyauterie.

\section{Le corps-outil du sujet-vivant}

Nous avons parlé de la corporéité factice des monades techniciennes, il convient à présent d'examiner le revers de cette problématique. Car si les sujets-vivants sont particulièrement résilients chez Kubrick, ils n'en sont pas moins victimes de leur dépendance à la Technique. La mécanisation des corps est un thème majeur du cinéma de Kubrick. Cela commence dès le début de son œuvre. Le premier opus du cinéaste, Fear \& Desire (1953), est une œuvre programmatique où la réflexion de Kubrick sur la guerre et la violence se révèle indissociable de son approche physiologique des affects et des pulsions. On sait que Kubrick chercha à faire disparaître ce film qu'il jugeait trop didactique et maladroit. Il s'agit pourtant d'une œuvre matricielle où l'équation fondamentale est déjà posée : Peur + Désir $=$ Folie.

Les protagonistes du film sont des soldats rescapés d'un accident d'avion qui se retrouvent dans une forêt à une dizaine de kilomètres des lignes de l'armée ennemie. Ils rencontrent une femme qui ne parle pas leur langue. Craignant qu'elle ne trahisse leur position, ils la ligotent à un arbre. Sydney, le jeune soldat le plus compatissant envers la jeune fille, se retrouve alors seul avec elle. Un désir sexuel attise la concupiscence du jeune soldat autant qu'un besoin de réconfort, un besoin compulsif de se faire materner. Le jeune Sydney se lance alors dans une pantomime grotesque pour faire rire la jeune fille. Et, à l'instar de Pyle dans Full Metal Jacket ou de Jack dans Shining, il sombre dans la folie parce qu'il ne peut se résoudre à commettre un viol : la frustration et la peur engendrent la folie. Eros et Thanatos, la pulsion vitale et la pulsion morbide, le désir (desire) et la peur (fear), sont les deux intensités d'affects qui 
traversent le sujet-vivant kubrickien, provoquant souvent un court-circuit qui se traduit par un dérèglement généralisé.

Chez Kubrick, la guerre n'instrumentalise pas la Technique pour devenir plus efficiente, car la Guerre et la Technique sont en fait co-originaires. Le premier outil est aussi la première arme. Le premier geste technique de l'homme est aussi le premier geste guerrier. Le premier meurtre est le point de départ de l'Histoire humaine. Il est important de souligner à quel point la violence et la guerre ont partie liée avec la Technique pour appréhender l'équation fondamentale : Peur + Désir = Folie. Qu'est-ce que la Peur ? Ce n'est pas simplement la « peur de mourir » du troufion qui est envoyé à l'abattoir, c'est, plus fondamentalement, la peur panique de Sydney devant sa prisonnière, celle d'être submergé par un désir qui aura tôt fait d'être métabolisé en violence.

Et qu'est-ce que le Désir? Ce ne sont donc pas simplement les " passions » en général, ce sont les pulsions, les affects qui traversent le corps. La Technique, quant à elle, ne doit donc pas être envisagée chez Kubrick comme l'émanation abstraite d'un " monde-cerveau ", mais comme un dispositif d'amplification et de contrôle des affects qui traversent le corps. De sorte que la Guerre - en tant qu'émanation de la Technique - ne serait, en définitive, rien d'autre qu'une forme de domestication des sujets-vivants par le truchement d'outils meurtriers et de procédures techniciennes de canalisation des affects.

On se souvient de la fameuse séquence des Sentiers de la gloire où le Colonel Dax, incarné par Kirk Douglas, se prépare à donner l'assaut à la Cote 110, "Anthill ", la "Fourmilière ». Dax passe en revue ses hommes épuisés avant de mener l'attaque... Dans les tranchées, Kubrick utilise ce qui deviendra sa figure de style de prédilection : un travelling-arrière raccordé à un "contre " travelling-avant alterné dans l'axe pour mettre en image la pulsion cinétique qui s'est emparé de Dax. Et nous assistons en direct à la mécanisation du corps de Dax : un " corps-outil " programmé pour mener ses hommes au carnage. Et justement, Dax passera tout le reste du film à tenter de sauver ces soldats d'un procès inique, à tenter de reprendre le contrôle de son corps, à tenter de contrer ce travelling arrière, cette impulsion cinétique qui l'aimante vers le champ de bataille et le transforme en une machine de guerre aveugle. Dax lutte pour que la Volonté de Puissance qui le traverse ne se transforme pas en Volonté de Néant. Mais il est déjà trop tard : il a déjà envoyé les soldats au carnage, l'énergie cinétique impulsée par ses coups de sifflet a été transmise, comme par contamination, à tout son régiment.

On pourrait croire que cette entreprise collective de mécanisation des corps n'est à l'œuvre que dans les guerres modernes, celles du $\mathrm{XX}^{\mathrm{e}}$ siècle qui sont dépeintes dans Fear \& Desire, Path of Glory ou Full Metal Jacket. Mais il suffit de s'intéresser à Barry Lyndon pour se rendre compte qu'il n'en est rien. On y voit des murailles de soldats marchant vers l'une vers l'autre comme des soldats de plomb. Ces petites mécaniques industrieuses sont synchronisées 
au son des fifres et des tambours. Ces "soldats de Vaucanson " sont déjà des "corps-machines" habités par la Volonté de Néant. Le dressage machinique des corps est donc déjà à l'œuvre au XVIII ${ }^{\mathrm{e}}$ siècle. Et Kubrick, même s'il ne fait jamais directement allusion à la philosophie des Lumières dans son film, ne se prive pas de nous offrir une mise en cause cinglante et ironique de l'utopie du progrès moral qu'a voulu prophétiser ce courant de pensée à travers toute l'Europe.

\section{Les procédures techniciennes et la volonté de néant}

Nous l'avons déjà souligné, chez Kubrick, la Guerre prend la forme d'une domestication des sujets-vivants par le truchement d'outils meurtriers et de procédures techniciennes de canalisation des affects. Des procédures techniciennes qu'il ne faudrait pas réduire à une simple confrontation guerrière sur le théâtre des opérations. Car ce sont, aussi, par exemple, les sermons incendiaires du sergent instructeur Hartman de Full Metal Jacket, cet « expert en intimidation " qui programme les jeunes recrues. De fait, le langage qu' utilise Hartman n'est pas simplement destiné à leur imposer une discipline, il sert aussi à « euphémiser » la violence, c'est-à-dire à la déréaliser. En se conformant à ce langage, qui rappelle aussi le "nadsat " composite d'Orange Mécanique, les Marines deviendront eux-mêmes des armes-vivantes, de véritables " corpsoutils ». Et l'on sait où ce processus d'intériorisation de la culpabilisation et de la dévalorisation de soi conduira Pyle.

Dans 2001, le langage est également envisagé comme une arme-outil, au même titre que pouvait l'être l'os des premiers hominidés. On se souvient des discussions entre les scientifiques russes et le docteur Heywood Floyd, ce fonctionnaire de la Technique, maniant parfaitement la langue de bois. Kubrick prend bien soin de lier, dans cette conversation entre les représentants des deux grandes puissances, la problématique de la Technique à celle de la guerre. L'enjeu n'est plus la prise de possession d'un territoire - la mare des hommessinges - mais celui d'un secret d'État. Mais il s'agit toujours de l'affrontement de deux tribus. Le changement d'échelle étend le conflit aux dimensions de la planète entière. Et, si toute forme de violence physique semble avoir été bannie de la société future, c'est toujours la même entreprise de domination qui se perpétue derrière les formules de politesse, les questions inquisitrices et les réponses évasives. Le "salon de guerre orbital » est une métaphore à peine voilée de la guerre froide.

Dans Folamour, le langage a également été perverti par l'homme de la Technique. Lorsque le colonel Mandrake reçoit l'ordre de Ripper, il ne mesure pas les conséquences induites par le " déclenchement du plan R ». Mandrake s'exécutera avant de réaliser avec horreur que le simple code dissimule, en réalité, la procédure destinée à annihiler l'humanité. Le langage codé 
induit une perte de réalité. La codification, la procédure, l'enchaînement des opérations techniques transforment les individus en simples rouages d'une mécanique qui les dépasse. Cependant, il ne faut pas perdre de vue que les procédures techniciennes du Docteur Folamour ne constituent pas une interface entre le monde et un énigmatique cerveau, une entité panoptique et abstraite telle que la conçoit Deleuze. Ces procédures techniciennes ne font que relayer la Volonté de Néant d'un individu singulièrement dérangé, elles deviennent la chambre d'écho de la frustration sexuelle du général Jack D. Ripper qui dissimule son impuissance en accusant les communistes de vouloir empoisonner les "fluides vitaux des Américains".

C'est donc un corps individuel, frustré et gorgé de ressentiment, qui est la véritable cause de l'Apocalypse. Et ce sera aussi un corps malade, entravé et libidineux, celui du Docteur Folamour, qui proposera d'édifier une nouvelle société dystopique et eugéniste après l'Apocalypse. Kubrick ne perd jamais de vue que la Volonté de Néant déployée par le dispositif technique s'origine dans des corps. La Technique conditionne le sujet-vivant, mais ce sont, en dernier ressort, les affects et les pulsions du corps qui s'expriment à travers elle. Il nous faut toutefois remarquer que le conditionnement du sujet-vivant par des procédures techniciennes abstraites - celles du langage formaté ou des codes n'engendre pas toujours nécessairement une Volonté de Néant. Il peut aussi engendrer un Néant de Volonté.

Ainsi, dans 2001, la Technique engendre une jouissance plate et confortable tout en neutralisant les angoisses. C'est l'ère de l'indifférence, de l'absence apathique des sens, de la fuite éperdue devant la mort mais, en aucun cas, celle d'une quelconque détresse métaphysique. Les hommes du futur sont décrits comme des cobayes de laboratoire effectuant leurs tours dans une grande roue. Ils se comportent comme des machines en effectuant leurs manœuvres routinières avec froideur et efficacité, en neutralisant leurs affects. La Technique, prothèse de nos corps et expression pervertie de notre Volonté de Puissance, produit des sujet-vivants désincarnés et trop faibles même pour le désespoir. Des "corps-outils " dont le Néant de Volonté trahit une profonde désaffection ontologique que l'on pourrait rapprocher de la « détresse de l'absence de détresse » diagnostiquée par Heidegger.

Tachons, à présent, de nous demander en quoi consiste l'approche physiologique de Kubrick et comment il parvient à rendre compte de la complexité des processus de résilience qui travaillent le sujet-vivant? Demandons-nous aussi comment ces processus résistent à ceux qui lui sont imposés par la civilisation technicienne? 


\section{L'approche physiologique: métabolisation de la volonté de puissance}

\section{Le Traitement Ludovico}

Les procédures techniciennes - ces dispositifs d'amplification et de contrôle des affects qui traversent les corps - sont un formidable moyen de domestication du sujet-vivant. Et la procédure technicienne la plus spectaculaire du cinéma de Kubrick est sans doute le Traitement Ludovico : cette méthode de conditionnement pavlovien imposée par les scientifiques d'Orange Mécanique au jeune délinquant Alex DeLarge. La force vitale qui s'exprime à travers Alex, est si débridée qu'elle ne pourra être interrompue que de manière artificielle et brutale. Car, en temps normal, cette force vitale ne s'accorde qu'avec la volonté de néant et ne connait aucun répit.

La Volonté de Néant d'Alex est incapable de lucidité et d'autocritique, elle est absolument tautologique et exprime la tendance fondamentale du déshérité nietzschéen : celle de vouloir le rien plutôt que de ne rien vouloir. De l'ultraviolence d'Alex - que l'on peut aussi interpréter comme surexposition volontaire aux excitations du dehors - résulte une intoxication des sens qui ne permet aucune évolution. Dans Orange mécanique, la notion d'excitation joue un rôle prépondérant : plutôt que de soigner le jeune délinquant en ayant recours à des méthodes apaisantes, les scientifiques capitalisent sur son avidité prédatrice et sa prodigieuse capacité à accueillir les excitations du dehors.

La Méthode Ludovico repose en effet sur le principe de la réversibilité des interprétations que produit l'intellect à partir de l'excitation. Je précise que j'entends ici par interprétation l'association entre une excitation et un état du corps positif ou négatif, c'est-à-dire du plaisir ou de la douleur. La méthode de conditionnement Ludovico dissocie les excitations ressenties à l'écoute de la Neuvième Symphonie de la joie qu'elle procure ordinairement à Alex. Les scientifiques l'associent artificiellement à du déplaisir créant ainsi un mal-être physiologique. Le jeune cobaye devient alors l'esclave de l'interprétation active de la Neuvième produite par son corps. En d'autres termes, la procédure technicienne "Ludovico » utilise les ressources interprétatives du sujet-vivant pour transformer l'avidité destructrice d'Alex, sa Volonté de Néant, en un Néant de Volonté.

Cependant Kubrick ne sous-estime pas la plasticité du sujet-vivant et sa faculté à s'autoréguler. Alex finira par se guérir lui-même du conditionnement : à la fin de son périple, les excitations produites par l'ultraviolence et la Neuvième seront de nouveau ressenties et interprétées comme des sources de plaisir. Comme dans Full Metal Jacket, 2001 ou Docteur Folamour, Kubrick met l'accent, dans Orange mécanique, sur cet élément irrationnel impossible à circonvenir qui distingue l'homme de la machine. Alex va guérir mais, curieusement, sa guérison n'adviendra que pendant sa convalescence après son suicide raté et un long calvaire parsemé de déconvenues, de brimades et de mauvais traitements. 
Comment expliquer cette prodigieuse résilience du sujet-vivant et sa "guérison»- qu'il ne faut, bien entendu, pas entendre dans un sens moral, mais strictement physiologique ? Comment expliquer que le long calvaire qu'endure Alex après avoir été « rectifié " par le traitement Ludovico, constitue un puissant contrepoison, un antidote radical à son conditionnement ?

Le processus de guérison : résilience du sujet-vivant (Orange Mécanique/ Docteur Folamour)

Dans Orange Mécanique, il apparaît clairement que les processus internes $\mathrm{du}$ sujet-vivant refusent de se voir assigner des fonctions arbitraires. Les nouvelles associations artificiellement créées par les scientifiques ne se maintiennent qu'un temps. Il y a, chez Kubrick, cette idée d'une identité propre au sujet-vivant qui, parce qu'elle est ouverte et en perpétuelle évolution, a les moyens d'échapper à une sclérose de la volition.

Incapable de se fermer aux blessures du dehors, l'Alex post-Ludovico ne cesse de remettre en question sa pluralité interne. Sa force d'assimilation est démesurément accrue, au point de n'être plus sélective. Durant toute la seconde partie du film, Alex se contente de recevoir passivement des douleurs qu'il ne parvient plus à réinterpréter. Or c'est paradoxalement en demeurant ouvert aux blessures du dehors que le sujet-vivant Alex va restaurer sa Volonté de Puissance. Ce qui, en lui, résiste au traitement Ludovico - le donné résiduel de sa mémoire corporelle - va faire ressurgir les anciennes équivalences entre les excitations $d u$ dehors et leur interprétation.

Ce processus d'auto-guérison nous permet de mieux comprendre ce qui, dans l'œuvre de Kubrick, différencie l'homme de la machine. La machine est un système rétroactiffermé sur lui-même se bornant à interpréter les excitations venues du dehors selon des schémas immuables. À l'inverse, le sujet-vivant est un ensemble de processus ouverts dont la pluralité interne est constamment remise en question, mais qui dispose de ressources sans cesse renouvelées pour faire face à l'adversité.

Voilà pourquoi la procédure technicienne "Ludovico " sera toujours condamnée à l'échec : son analyse du sujet-vivant est erronée. De fait, les procédures techniciennes ne peuvent jamais excéder les facultés régénératrices du sujet-vivant. Et si l'Orange-Mécanique du titre est une manière d'affirmer que le devenir-machine de l'homme est une tendance irrépressible du monde moderne, il apparait clairement que cette tendance est vouée à l'échec. L'analyse du sujet-vivant entreprise par Kubrick s'origine dans une compréhension physiologique des facultés de résistance du vivant. L'incroyable plasticité de son "sujet-vivant " ne peut être appréhendée qu'à travers l'approche généalogique des représentations. Elle est plurielle, dynamique et discontinue: celle, incarnée et changeante, du corps-sujet nietzschéen plutôt que celle, glaciale et abstraite, de la raison transcendantale kantienne. 
Le Docteur Folamour, lui-même, ce pur produit de la civilisation technicienne, cet être hybride - mi-homme, mi-machine - est traversé par une Volonté de Puissance qui surpasse sa Volonté de Néant. Alors que le monde est sur le point d'être détruit, le corps-machinique du funeste docteur se lève de son fauteuil roulant dans un ultime réflexe érectile qui annonce le grand orgasme nucléaire à venir. La main-prothèse qui menaçait d'étrangler Folamour quelques instants plutôt, laisse place aux incroyables capacités d'auto-guérison du corps. Le fameux "Mein Fuhrer, I can walk! » signifie que l'irrationalité s'est enfin libérée de sa gangue rationnelle. Sous les procédures cryptiques et aseptisées de la Technique, ce sont les affects et les pulsions du sujet-vivant qui refont surface.

Certes, la guérison physique de Folamour, le plus radical des nihilistes kubrickiens, est associée à la plus radicale des procédures techniciennes : l'holocauste nucléaire. Cependant n'oublions pas que Folamour n'envisage pas l'Apocalypse comme une catastrophe mais comme l'occasion d'une nouvelle renaissance: la possibilité de régénérer la société des hommes, cet organisme paralytique et malade. De sorte que la guérison miraculeuse de Folamour nous apparaît aussi bien comme une parfaite allégorie de la Volonté de Néant à l'œuvre dans le processus techniciste que comme une parfaite incarnation de la Volonté de Puissance qui traverse le sujet-vivant.

\section{L'œil : machine de vision (Orange Mécanique/EWS/Shining)}

Il nous faut encore dire un mot sur l'organe qui, chez Kubrick, permet d'appréhender le "sujet-vivant " d'un point de vue physiologique. Cet organe, c'est bien évidemment l'œil. Car, chez Kubrick, c'est par le regard que transitent les affects et les pulsions. Souvenons-nous du regard dentelé et prédateur d'Alex cherchant à satisfaire une pulsion scopique. L'acuité criminelle du regard d'Alex est, dans une certaine mesure, comparable à celle du primate de 2001 lorsque ce dernier prend soudain conscience du pouvoir destructeur de l'arme-outil. Le regard d'Alex, regard calculateur de son propre plaisir et dénué de compassion, est, d'une certaine façon, déjà lié à la Technique. Pour que les pulsions violentes d'Alex s'actualisent, il faut que sa vision se mécanise, devienne elle-même une arme.

En définitive, la pulsion scopique, active et sadique d'Alex sera également celle du spectateur. Et c'est en suscitant l'adhésion (inconsciente) du spectateur devant le spectacle des horreurs ("horrorshow») que Kubrick parvient à mettre au jour la véritable nature de la pulsion scopique. La machine de vision humaine est un outil mis à la disposition des hommes par l'évolution. Avide de violence, elle participe de la même logique prédatrice que celle du monde de la Technique. L'œil humain est une machine-vivante qui ne se contente pas de recueillir passivement les données du sensible : il les recycle activement afin d'adapter le réel à ses besoins. 
Dans 2001, la circulation des images - images mentales/images réelles - est le moyen par lequel le cinéaste signifie le passage de l'animalité à l'humanité. L'image mentale imprimée par le monolithe dans l'esprit du proto-humain permet la transformation d'un os en outil. Issu d'une autre temporalité, le monolithe transmet aux premiers hommes le principe du mouvement des astres et actualise une potentialité de leur esprit. Cette potentialité - qu'Aristote appelait l'imagination délibérative - permet à l'homme de ne plus vivre que dans l'instant, mais de se projeter dans le temps.

Cela nous est suggéré lors de la fameuse séquence où après s'être souvenu $\mathrm{du}$ monolithe, l'homme-singe procède à une association d'idées : il voit un phacochère s'effondrer au ralenti tout en fracassant des squelettes avec son os-arme. L'image de l'animal terrassé est à la fois la projection d'un fantasme et la résolution d'une délibération. L'œil humain est donc à la fois organique et intérieur: il fait partie du corps, mais s'apparente aussi à une machine. Et cette symbiose corps/machine le cinéaste ne cessera de l'approfondir dans son ouvre.

Car ce n'est pas seulement dans 2001 et Orange mécanique que ce lien entre la vision, la Technique et le corps est mis en image par Kubrick. Dans Eyes Wide Shut, le regard occupe, comme le titre l'indique, une fois de plus un rôle central. Plus que jamais, il est placé sous le signe de l'ambivalence et de la contradiction. Là aussi, le regard est un passage vers l'intériorité, un moyen d'accéder à une connaissance non verbale, à un donné brut qui excède parfois ce que l'entendement humain peut soutenir. Parce qu'il appartient au domaine du fantasme, le récit d'Alice dans Eyes Wide Shut contamine l'imaginaire de Bill qui le recycle en une série de vignettes érotiques en mouvement. Le cerveau de Bill devient alors une camera oscura miniature où se projettent le ressentiment, la frustration et l'amertume d'un mari virtuellement cocufié.

Les flashs qui ponctuent le parcours de Bill lors de son errance nocturne sont des images fixes, des images purement mentales. Bill, devenu une véritable " machine de vision ", tente d'objectiver le fantasme de sa femme, de le convertir en stimuli visuels, pour lui conférer le surplus de réalité qui lui manquait. Mais la mise en image échoue lamentablement car Bill, à la différence de sa femme, est plus un voyeur qu'un metteur en scène.

À ce stade, il est intéressant de remarquer que les lents zooms avant qu'utilise Kubrick pour nous présenter l'image mentale où Bill voit sa femme s'abandonner au marin, sont similaires à ceux du "shining ". Il s'agit, pour Kubrick, de nous donner à voir dans les deux films, une "image-corps ", c'est-à-dire une vision métabolisée par des sujets-vivants en proie à un intense tourment intérieur. Dans Eyes Wide Shut, Bill, hanté par le désir d'Alice, cherche - en vain - à reprendre possession de lui-même. Dans Shining, Danny et Jack sont traversés par un flux parasitaire d'images morbides.

Mais, tandis Danny résiste, Jack s'abandonne aux images parasitaires et succombe à la pulsion scopique. L'écrivain raté métabolise des visions malsaines 
et se laisse investir par le récit d'un autre. L'entité maléfique de l'Overlook - le regard surplombant - le submerge et l'absorbe. Dès lors, le regard de Jack ne se contente pas d'être un regard prédateur, il devient cannibale: c'est le regard d'un père saturnien qui veut manger son fils, celui d'un extra-lucide qui ne peut se délivrer de sa terreur quén la réalisant. Car la seule façon d'annuler la vision, c'est de la réaliser.

On vient de le voir, la métabolisation des images par la machine de vision humaine si elle peut conduire au triomphe de l'intelligence et des affects comme dans Orange Mécanique et dans 2001, peut aussi devenir un piège hypnotique qui prive le sujet-vivant de son intégrité physiologique. Chez Kubrick, le corps est, avant tout, un oil avide qui absorbe et qui recycle les images.

\section{La Mètis : l'intelligence conjecturale du corps}

Après nous être intéressé à l'approche physiologique de Kubrick, à sa compréhension du sujet vivant comme entité plurielle, dynamique, discontinue, il convient d'insister sur le fait que la prodigieuse plasticité, la fantastique résilience $\mathrm{du}$ sujet-vivant n'est pas la seule caractéristique des corps kubrickiens. Loin de se limiter à une vision binaire et dualiste des rapports entre le corps et l'esprit, Kubrick n'envisage jamais l'un sans l'autre. C'est parce que, dans certains cas, il existe une étroite connivence entre le corps et l'esprit que le cinéaste envisage aussi la possibilité qui est donnée à l'homme de se surpasser. Cela est particulièrement évident dans trois de ses films : 2001, Shining et Eyes Wide Shut.

\section{1 : le Cyclope de la Technique}

Dans 2001, ce n'est ni une forme de religiosité, ni une aptitude particulière à endurer la souffrance qui permet de surmonter le nihilisme technologique. C'est un retour à quelque chose de plus fondamentalement humain. De quelle manière Bowman - l'homme-arc, l'homme-pont - parvient-il à vaincre $\mathrm{Hal}$, le cyclope de la Technique? L'homme peut supplanter la machine parce qu'il est un sujet-vivant, une pluralité ouverte en perpétuelle évolution. Hal, quant à lui, est un esprit désincarné dont le corps est un os flottant dans le vide spatial, un corps devenu un tombeau : le Séma Soma de Platon.

C'est pourquoi le corps de Bowman qui, paradoxalement, est l'indice de sa finitude, est également le supplément d'infini qui lui permettra de franchir la porte des étoiles et d'accomplir sa transmutation en foetus astral. La machine a l'avantage de la raison calculante. Bowman va pourtant réussir à la vaincre en utilisant une ruse : il s'expulse du Pod dans le vide spatial sans son casque. C'est ce coup imprévisible qui va mettre l'ordinateur échec et mat.

Cette ruse, les Grecs l'appelaient la Métis, une intelligence pragmatique liée à la faculté d'improviser, à profiter du moment opportun. La stratégie inattendue de Bowman renvoie d'ailleurs aux grandes épopées de la Grèce 
antique comme celle des Argonautes qui, en échange d'un trépied destiné à Delphes, obtinrent du dieu Triton qu'il leur montre le chenal pour sortir des bas-fonds. Ce que Triton révèle aux voyageurs réduits à l'aporie, est un poros (ou diekplous), une manœuvre maritime consistant en un brusque volte-face destinée à laisser l'ennemi désemparé.

La Métis est, en effet, liée à une certaine audace : celle qui parvient à déterminer le kairos, le moment opportun. La sortie de Bowman dans le vide interstellaire exprime cette audace qui implique, pour les anciens Grecs, une connaissance intuitive assujettie à la contingence et à la corporéité. L'homme dont l'action est tendue vers une fin, doit toujours tenir compte de sa propre finitude - de son corps - et savoir que son action s'exerce dans un domaine ò̀ rien n'est jamais stable, le domaine de la contingence. Si l'intelligence humaine est associée à la Métis, c'est le statut d'être raisonnable de l'homme qui risque d'être assimilé à celui de l'animal, au vivant sans logos: l'homme ne serait, dès lors, rien d'autre qu'un animal plus rusé que les autres.

Or c'est précisément sur ce postulat que repose le propos philosophique implicite de 2001. L'homme n'y est pas envisagé comme un être radicalement différencié de l'animal, mais comme un animal doué d'une ruse si puissante qu'elle lui permet de transformer son environnement pour assurer sa survie. C'est également pour assurer sa survie que Bowman a recours à la Métis. La singularité et la profonde modernité de 2001 découlent de cette conception particulière de l'intelligence humaine. Le nihilisme de l'ère de la Technique, incarné par Hal, se révèle impuissant parce qu'en dépit de ses formidables potentialités, son intelligence calculante est une intelligence orpheline d'un corps.

C’est bien là le drame de $\mathrm{Hal}$ : être une conscience privée de corps qui se rattache désespérément à sa mission pour se sentir exister. C'est la raison pour laquelle l'humanité de Hal n'était pas une condition suffisante pour remporter la victoire dans le combat qui l'opposait à Bowman et gagner le droit d'atteindre un nouveau palier évolutif. Quant au voyage de Bowman au-delà des étoiles, il présente également des similitudes avec l'univers sémantique et visuel de la Métis.

Lors de sa course folle à travers le tunnel de lumière, Bowman voit sous ses yeux se transformer l'espace glacial, ténébreux et infini en une route balisée par d'énigmatiques signaux. En présence de ses compagnons, Jason adresse une prière solennelle à Apollon afin qu'il lui indique les voies de passages (poroi). C'est Apollon qui transforme la "mer comme étendue abyssale, chaotique, veuve de routes" en une voie d'accès praticable. Dans 2001, c'est le monolithe qui ouvre, dans l'espace infranchissable (apeiros), le couloir entre les pierres errantes et les sémaphores qui conduisent Bowman « au-delà de l'infini ». 


\section{Shining : le Minotaure et l'enfant}

Dany, l'enfant doué du shining, fait lui aussi usage de sa Métis : on se souvient de la manière dont il échappe à son père-minotaure en marchant à reculons dans les traces de ses pas imprimées dans la neige. La séquence a lieu dans labyrinthe végétal, un lieu où les chemins qui bifurquent ne mènent souvent nulle part, une construction qui établit le lien entre un espace concret et un espace mental, un espace où le temps n'est plus linéaire mais circulaire.

Comment Danny parvient-il à s'en échapper ? L'enfant est pourvu d'un esprit de débrouillardise et d'une grande mobilité. Cet esprit de débrouillardise, les Grecs l'appellent "purpalàmès " et l'associent à Hermès de la Mètis : "le purpalàmes est un madré, un poikilos, un individu qui comprend d'un coup et qui, en un tour de main, invente une combine : il est vif comme le feu. ${ }^{11}$

Dans l'hymne homérique, on raconte qu'Hermès a fait disparaitre le troupeau de bœufs de son frère Apollon au cours de la nuit. Pour faire disparaitre la marque des sabots des animaux, Hermès va déployer ses talents de ruse. Pour brouiller les pistes, il opère un renversement des traces. Les vaches s'avancent à reculons, la tête tournée vers leur bouvier. Hermès chemine, tête tournée vers ses bêtes et pieds inversés. Les traces qu'il imprime sur le sol conduisent dans la direction opposée à celle qu'aurait dû prendre le troupeau volé. Ce renversement provoque la panique des limiers lancés par Apollon sur la piste du voleur. Ils découvrent soudainement que «ce qui allait en avant, va en arrière" et que "les contraires s'entrelacent les uns devant les autres " ${ }^{12}$.

La ruse de Danny relève d'un principe de machination similaire à celui d'Hermès. C'est une ruse bien connue des Anciens Grecs que Xénophon appelle aussi la " ruse du lièvre " : elle consiste, lors d'une chasse, à " doubler la voie ", c'est-à-dire à revenir sur ses pas pour mettre les chiens en défaut ${ }^{13}$. Aux pièges circulaires et régressifs de l'Overlook répond la Métis créatrice de Danny, une intelligence chevillée au corps et résolument tournée vers l'avenir. C'est d'ailleurs parce qu'il est capable de saisir l'instant présent, le moment opportun (kairos) que Danny parvient à vaincre l'Overlook. La manœuvre de Danny consiste à résister au recourbement du temps, à contrecarrer le piège spatial et temporel qui lui est tendu par l'Overlook. Jack, incapable d'un tel retournement, poursuit la métamorphose et régresse jusqu'à l'animalité. Vaincu par l'« enfant lumière »- ce nouveau Thésée -, le Minotaure Jack s'enlise dans une éternité cauchemardesque, avec le souffle court d'un taureau étrillé.

11 M. Detienne et J.-P. Vernant, Les Ruses de l'intelligence, La métis des Grecs, Paris, Flammarion, 1974, p. 266.

12 Ibid., p. 267.

13 Cf. Xénophon, L'Art de la chasse, VI. 21, Delebecque (p. 76, nº 1). 


\section{Eyes Wide Shut : la maïutique dionysiaque}

Eyes Wide Shut est, en un sens, une autre histoire de labyrinthe. Ce film raconte l'odyssée intérieure d'un homme qui chemine à travers un labyrinthe de fantasmes et prend peu à peu conscience de ses limites. C'est la Mètis de sa femme qui lui permettra d'en trouver la sortie et de se réconcilier avec elle. Dans la dernière scène qui a lieu dans un magasin de jouets, Alice oppose à l'ultime proposition théorique de Bill - il projette leur relation dans l'éternité en employant le mot "forever" -, une proposition simple et abrupte : "Just fuck ". En choisissant de conclure son film, non sur la traditionnelle scène de réconciliation propre aux comédies du remariage mais en insistant, au contraire, sur le caractère à la fois contingent et réfléchi de la décision qui va précéder l'union des époux, Kubrick rend hommage à la Mètis d'Alice.

Il rend aussi hommage à une nouvelle génération de femmes émancipées de l'ancien ordre patriarcal qui choisissent de mettre leur Mètis au service du vouloir-vivre et de l'affirmation du devenir plutôt que de l'abandonner au nihilisme du monde de la Technique. Le trivial "Just fuck" est un appel à un retour à l'ordinaire, à une reconnaissance (acknowledgement) de notre condition sceptique, au sens où l'entend Stanley Cavell. Finalement, il n'y a peut-être pas besoin d'être un surhomme pour devenir un affirmateur $d u$ vouloir-vivre, semble vouloir nous dire Kubrick, il suffit peut-être d'accepter d'être simplement humain.

Eyes Wide Shut nous confronte à deux manières d'être au monde radicalement opposées : celle de Bill, en apparence posée et rationnelle mais en réalité superficielle et narcissique et celle d'Alice, intuitive mais cohérente. Dans la fameuse scène du joint, tout semble indiquer la crispation de Bill, pour ne pas dire sa frustration : dès que l'objet de son désir lui échappe, il adopte une posture hiératique qui ne variera pas pendant toute la durée de la dispute. Bill tente de garder le contrôle de son corps à l'inverse d'Alice qui n'hésite pas à changer de position, à se lever, à déambuler dans la pièce ou à s'asseoir par terre. Alice s'exprime aussi bien avec des mots qu'avec des gestes et des mouvements. Elle ne soustrait pas son corps de la dynamique de son raisonnement: elle fait parler son corps.

Loin d'être déconstruit ou aléatoire, l'argumentaire d'Alice - quoique échappant à l'ordre du rationnel - obéit à une logique rigoureuse, sorte de maïentique intuitive où l'ironie est utilisée pour faire glisser le discours vers les sentiments et le vécu personnel.

Alice se moque ouvertement de Bill, le provoque dans une joute verbale et l'oblige à abandonner son rôle de docteur et de mari pour redevenir un homme générique. La Métis d'Alice est à l'œuvre : son acuité intellectuelle évoque la " pénétration d'esprit ", cette qualité de l'intelligence dont Aristote crédite surtout la sage-femme qui ne se trompe pas sur le but à atteindre et anticipe intuitivement le déroulement de chacune des étapes risquées de la parturition. 
Sous sa forme positive, ce type d'intelligence sert également à désigner la "justesse de coup d'œil " (eustochia) ${ }^{14}$ combinant à la fois l'idée d'une visée, d'un but à atteindre et d'un regard aquilin qui s'oppose radicalement à la myopie existentielle de Bill. Prendre pour cible se dit d'ailleurs en grec stochazesthai, un verbe qui "appartient au vocabulaire de l'archer et du chasseur. " ${ }^{15}$ Bill est impuissant face à la Métis d'Alice, son discours va s'emballer, devenir agressif, incapable d'autre chose que de répétition jusqu'à se taire pour ne laisser apparaître qu'un corps athlétique mais rigidifié dans une attitude de passivité absolue.

Le rire d'Alice est dès lors une manifestation de sa supériorité, il résonne comme un vertere pollicem (pouce vers le bas) dans cette chambre conjugale qui, à l'occasion, s'est transformée en arène des sexes. Le monologue d'Alice qui clôt la scène du joint est un récit intimiste plutôt que didactique. Il ouvrira la faille qui absorbera l'imaginaire appauvri de Bill et déclenchera, par la suite, ses visions.

Le langage d'Alice appartient à un registre spécifique de l'intelligence qui ne se confond pas avec une activité rationnelle : pour Aristote, la Métis s'efforce, en tâtonnant et par conjecture, d'atteindre le but visé et, se faisant, relève "d'un mode extérieur à l'epistémé, au savoir " "16. N'oublions pas que la Métis est aussi une déité marine dans les théogonies orphiques, une déité qui se confronte à une réalité multiple, changeante et dont le pouvoir de polymorphie est illimité. La Métis est en contact avec les puissances de l'inconscient et, dès lors, on ne s'étonnera pas qu'Alice, sans rien savoir des pérégrinations de son mari, ait été capable de les rêver en les transfigurant.

Pour finir, revenons un instant à la notion deleuzienne de "mondecerveau ". Nous l'avons vu, le corps, ses affects et ses pulsions, occupe une place centrale chez Kubrick. On pourrait penser, comme Deleuze, que les monades techniciennes, ces systèmes panoptiques et ubiquitaires, sont à l'origine des affects et des pulsions qui traversent les corps des sujet-vivants. Et, de fait, le dressage du "bétail " humain s'effectue par le biais de procédures techniciennes élaborées qui prennent des formes variées : outils meurtriers, discipline militaire, langage composite ou politicien, conditionnement psychosomatique, etc. Cependant, les monades techniciennes qui peuplent l'univers de Kubrick sont des machines dépourvues de corps et qui cherchent désespérément à un avoir un. Inversement, les sujets-vivants ont un corps qu'ils oublient pour s'harmoniser aux procédures techniciennes et embrasser leur « devenir-machine».

14 M. Detienne et J.-P. Vernant, Les Ruses de l'intelligence, La métis des Grecs, Paris, Flammarion, 1974, p. 297.

15 Ibid.

16 Ibid., p. 10. 
Mais les processus par lesquels une telle métamorphose est possible sont envisagés par Kubrick de telle façon qu'il sera toujours impossible de réduire les sujets-vivants à des machines. D'abord parce que si la Volonté de Puissance peut effectivement être convertie en Volonté de Néant ou en Néant de Volonté par les procédures techniciennes, cette conversion sera toujours incomplète, partielle, inachevée. Les facultés de résistance des sujets-vivants sont insoupçonnées et immenses : Hal est vaincu par Bowman, Jack et l'Overlook par Danny, le "Traitement Ludivico " par Alex, le conditionnement militaire par Joker, et l'Apocalypse elle-même, par le sursaut érectile du Docteur Folamour.

Ensuite parce que la Technique ne peut être appréhendée indépendamment de l'hominisation, comme nous pouvons le voir dans 2001 et que le corps est lui-même, intrinsèquement et dès son origine, un produit de la procédure technicienne originelle : celle de l'évolution ou celle du bond évolutif induit par les énigmatiques monolithes. L'œil est l'outil-vivant grâce auquel le corps accède à l'intelligence et devient lui-même producteur de Technique. Il est la machine organique grâce à laquelle le sujet-vivant parvient à dominer son milieu, mais il est aussi la machine de vision grâce à laquelle Kubrick explore l'intériorité des sujet-vivants.

D'autre part, nous avons vu que l'attitude des sujets-vivants face au nihilisme technologique ne se limitait pas à faire montre de résilience. La Mètis est l'intelligence $d u$ corps, l'intelligence conjecturale grâce à laquelle les héros kubrickiens peuvent se surpasser et triompher du nihilisme de la civilisation technicienne. Pourtant la Mètis n'est pas étrangère à la Technique, elle en est même l'expression la plus noble.

\author{
Sam Azulys \\ New York University, Paris \\ samazulys@noos.fr
}

\title{
Résumé
}

Selon Gilles Deleuze, le cinéma de Kubrick est un "cinéma du cerveau " ${ }^{17}$ plutôt qu'un cinéma du corps, c'est-à-dire un cinéma où c'est d'abord et avant tout « le cerveau qui est mis en scène ${ }^{18}$. Penser les rapports entre le corps et l'esprit dans le cinéma de Kubrick nous paraît être un enjeu d'autant plus fondamental que son cinéma a longtemps été envisagé comme un « monde-cerveau » façonné par un démiurge perfectionniste jusqu’à l'obsession. Le «mondecerveau »- autre expression deleuzienne - de Kubrick semble pourtant toujours menacé par une entropie galopante qui, dans la plupart des cas, le fait, in fine, basculer dans le chaos.

17 Gilles Deleuze, L'Image-temps. Cinéma 2, Minuit, Paris, 1985, p. 267-268.

18 Ibid. 
Cependant, même si Kubrick adopte effectivement l'approche distanciée et analytique d'un diagnosticien soucieux de mettre au jour l'irrationalité pathologique d'un monde occidental en déroute, il nous semble accorder un profond intérêt à la question du corps. C'est pourquoi l'idée deleuzienne selon laquelle, dans l'œuvre de Kubrick, les attitudes du corps dépendent toujours du cerveau mérite, selon nous, d'être discutée. En effet, une approche dualiste et finalement réductrice ne semble pas rendre justice à la complexité des rapports qu'entretiennent le corps et l'esprit dans le cinéma de Kubrick.

Nous tenterons de montrer que le cinéaste, loin de s'en tenir à "l'identité du monde et du cerveau " comme le suggère Deleuze, propose une physiologie du sujet-vivant qui n'est pas si éloignée de l'approche nietzschéenne. Ainsi, lorsque les forces vitales se sont amenuisées, le néant de volonté peut se transformer en volonté de néant, c'est-à-dire en une volonté de puissance dévoyée, comme c'est par exemple le cas dans Docteur Folamour ou Orange Mécanique. Or, cette volonté de puissance est si débridée que même le nihilisme technologique triomphant ne parvient pas à la canaliser.

Dans l'œuvre du cinéaste, c'est donc en réalité le corps qui tient en échec le cerveau. Loin de nous proposer une vision pessimiste et dualiste de l'existence, Stanley Kubrick interroge le rapport du corps et de l'esprit pour réexaminer nos valeurs humanistes à la lumière des mutations technologiques et revaloriser la Mètis, cette intelligence pragmatique liée à la faculté d'improviser que les anciens Grecs identifiaient à la ruse et qui suppose l'existence d'une étroite connivence entre le corps et l'esprit.

\title{
Mots-clés
}

Kubrick, nihilisme, corps, esprit, Mètis, Nietzsche, Deleuze.

\begin{abstract}
According to Gilles Deleuze, Kubrick's cinema is "a cinema of the brain" rather than "a cinema of the body", which is to say a cinema in which it is first and foremost "the brain which is staged". Exploring the relation between brain and body in Kubrick's cinema seems all the more necessary since his work has long been considered as a "brain-world", shaped by an obsessively perfectionist demiurge. Such "brain-world"-another Deleuzian expression- yet appears to be threatened by a raving entropy which, in most cases, plunges the world into chaos.

However, even though Kubrick does adopt a distanciated, analytical approach akin to a diagnostician mindful to highlight the pathological irrationality of a Western world in turmoil, I argue he also pays careful attention to the body. That is why I believe the Deleuzian idea according to which in Kubrick's oeuvre, the body's reactions always depend on the brain, deserves to be questioned. A dualistic -and ultimately reductive- approach does not fully encompass the subtlety of the relations between body and mind that Kubrick depicts.

I therefore argue that the filmmaker, far from sticking to an "identity of world and brain" as Deleuze suggests, offers a physiology of the living subject reminiscent of the Nietzschean approach. Thus, when vital forces have diminished, the nullity of will can morph into a will to nothingness, that is to say a distorted will to power, as is the case in Doctor Strangelove or A Clockwork Orange. And yet, the will to power endures despite all the attempts to channel it through a triumphant technological nihilism.

In the filmmaker's work, it is thus the body which instead keeps the brain in check. Far from offering a pessimistic, dualistic view of life, Stanley Kubrick questions the relation between body and mind to better reexamine our humanist values in the light of technological mutations and ends up valuing Metis, this pragmatic intelligence connected to the faculty to improvise that ancient Greeks associated to ruse and which presupposes a tight connivance between body and mind.
\end{abstract}

Keywords

Kubrick, nihilism, body, mind, Mètis, Nietzsche, Deleuze. 


\section{NOTICE BIOGRAPHIQUE}

Sam Azulys est philosophe et professeur d'analyse filmique à l'Université de New York à Paris. Son ouvrage Stanley Kubrick: une odyssée philosophique (Éditions de la Transparence, 2011) se propose d'étudier les rapports entre le cinéaste et des philosophes comme Nietzsche, Heidegger ou Jünger. Il vient de publier Philosopher avec Game of Thrones aux Éditions Ellipses. 